University of Nebraska - Lincoln

DigitalCommons@University of Nebraska - Lincoln

Robert Katz Publications

Research Papers in Physics and Astronomy

August 1991

\title{
A Track Physics Model of Radiation Action
}

Robert Katz

University of Nebraska-Lincoln, rkatz2@unl.edu

Follow this and additional works at: https://digitalcommons.unl.edu/physicskatz

Part of the Physics Commons

Katz, Robert, "A Track Physics Model of Radiation Action" (1991). Robert Katz Publications. 78.

https://digitalcommons.unl.edu/physicskatz/78

This Article is brought to you for free and open access by the Research Papers in Physics and Astronomy at DigitalCommons@University of Nebraska - Lincoln. It has been accepted for inclusion in Robert Katz Publications by an authorized administrator of DigitalCommons@University of Nebraska - Lincoln. 
Published in Radiation Physics and Chemistry 37:1 (1991), pp. 125-129; formerly International Journal

of Radiation Applications and Instrumentation. Part C. Radiation Physics and Chemistry

http://www.sciencedirect.com/science/journal/0969806X

Copyright (C) 1991 Pergamon Press plc. Used by permission.

\title{
A Track Physics Model of Radiation Action
}

\author{
Robert Katz \\ University of Nebraska-Lincoln, Lincoln, NE 68588-0111, U.S.A.
}

\begin{abstract}
A model of radiation action by energetic heavy ions, inspired by a study of particle tracks in electron sensitive emulsion, has been successfully applied to many radiation effects in condensed matter, yielding quantitative descriptions and occasional predictions. Radiation effects are attributed primarily to secondary electrons. Each detector is imagined to be a collection of targets whose radiosensitivity is represented parametrically. We avoid a mechanistic description of detector response so as to retain the global character of the model. Attention is focused on the structure of particle tracks, on the radial deposition of dose about an ion's path by " $\delta$-rays," and on action cross sections.
\end{abstract}

\section{Introduction}

The analysis of experiments in atomic or nuclear physics in which beams of photons or charged particles are directed at targets have as their goal the determination of an interaction cross section as a function of projectile energy. The beam is monoenergetic, and is so disposed that the interactions are of single projectiles with single targets. From such experiments we cannot obtain more information than is contained in the cross section. This is often the case in radiation effects as well. One must restrain the impulse to overinterpret experimental findings.

There is a basic difference between the study of radiation effects and of basic physics, for in radiation studies the condensed matter medium is both a source of the secondary particles which are largely responsible for the observed effects, and the container of the affected targets. Both aspects of the irradiated medium must be taken into account in any model. Thus radiation experiments have additional complexity as compared to those in basic physics. Yet, where possible, the analysis of radiation experiments should yield an "action cross section." This means that we must consider the irradiated medium as an assemblage of targets, and that the action cross section represents the probability that the interaction of an incident projectile with the target ultimately results in the measured end point. We use the term "detector" to describe any substance in which an observable effect is produced by radiation.

Detectors in which the observed effect arises from the interaction of single electrons with isolated targets are called 1-hit detectors. Many radiation effects results from the interaction of several electrons with a target. These are many-hit detectors. Where an effect cannot be produced by single electrons incident on a target, it may still result from the action of the many clustered $\delta$-rays about the path of a single heavy ion. While cross section is an inappropriate description for electron irradiations for many hit detectors, it is nevertheless appropriate for heavy ion bombardment, for a single heavy ion produces the end point. For any radiation effect, we must know whether it can result from interaction with a single electron.

Irradiations with $\gamma$-rays or neutrons or with any "mixed" radiation environment are difficult to interpret. The response is frequently reported as a function of absorbed dose, impossible to relate to monoenergetic charged particle fluence. Dose is frequently an inadequate parameter for characterizing response. So also are amendments associated with particle energy, or restricted LET (linear energy transfer or stopping power restricted to within a small radial distance or to small energy transfers), or average LET, or the distribution of energy depositions in microscopic volumes. Radiation effects are not readily correlated to single parameter reductions of a radiation field. Indeed the properties of the radiation field and those of the medium are not separable variables, so that one cannot represent effects as a product of two factors, one of which represents the "radiation quality" while the other represents the irradiated medium.

To understand the effects of variations in "radiation quality" the radiation field is often decomposed into its constituent excitations and ionizations, or to energy deposition into appropriately small volumes, generally favored by those seeking mechanistic explanations, or into a collection of particle tracks, favored for parametric descriptions. Neither the constituent excitations and ionizations nor energy deposition in small volumes (ICRU, 1983) has led to a calculation of cross sections, or to an $a b$ initio calculation of radiation effects in condensed matter, however much this is desired. A decomposition into particle tracks coupled with a parametric description of the detector has had somewhat greater success.

\section{The Track Physics Model}

Our model is based on the assumption that the effects produced by secondary electrons from $\gamma$-rays and those 
from the secondary electrons from heavy ions ( $\delta$-rays) are comparable at the same "dose."

In this assumption we neglect any differences in the electron energy spectra, basing this neglect on the assumption that it is the electron slowing down spectrum rather than the initial electron energy spectrum which is responsible for radiation action (Hamm et al., 1978). When we speak of the dose of $\delta$-rays surrounding a heavy ion's path we imagine that we study the energy deposited in nests of coaxial cylindrical shells surrounding many ions. Thus the dose of $\delta$-rays within a shell is an average quantity, over a synthetic large volume made up of equivalent shells about many ions (Waligorski et al., 1986). We use the effect produced in a macroscopic volume by a given dose of $\gamma$-rays to estimate the effect produced in the shell about our typical, averaged ion. That effect is imagined to be the probability of activating a target as a function of macroscopic dose. Thus, even at large distances from an ion's path where there are very few $\delta$-rays penetrating a shell we estimate the effect on the basis of a similarly low dose of $\gamma$-rays where there also few secondary electrons. This perspective implies that the response to a dose of electrons, photons, or $\delta$-rays is nearly independent of the electron energy spectrum.

This approximation may not be realized in all detectors or for all initial energy spectra. In radiobiology orthovoltage $\mathrm{X}$-rays are thought to be somewhat (say $10 \%)$ more effective than $\gamma$-rays at the same dose, and some experiments indicate that the characteristic $\mathrm{X}$-rays from carbon are even more efficient (perhaps as much as $2 \times$ ), though this is somewhat controversial at present. The supralinearity in TLD-100 (LiF) is known to depend on the energy of incident X-rays (Suntharalingam and Cameron, 1969). The response of photographic emulsion displays absorption edges appropriate to its composition.

We also neglect the temporal differences between the duration of a $\gamma$-ray exposure and the extremely short pulse of $\delta$-rays which irradiates a target as the ion passes. For this we have no justification. This neglect of "dose rate," or of the differences in irradiation times must be reconsidered when theory and experiment diverge.

As models of this response we use formulations from biological target theory (Dertinger and Jung, 1970) called the multi-hit and multi-target models. For most physical detectors we use the multi-hit model based on the cumulative Poisson distribution. Here $C$ is the minimum number of hits per target to activate it, $A$ is the number of trials per target and $X$ is the number of hits per target. We have it that, after an average of $A$ trials per target, the probability that a target will experience $X$ hits is

$$
P(X, A)=A^{X} \mathrm{e}^{-A} / X !
$$

and the probability that a target will experience $C$ or more hits is

$$
P(C, A)=1-\sum_{X=0}^{X=C-1} P(X, A) .
$$

We typically use a multi-target model for biological cells, giving the probability that each of $m$ targets will each experience 1 or more hits, as

$$
P(m, A)=\left(1-\mathrm{e}^{-A}\right)^{m} .
$$

In these expressions, $A$ the average number of trials per target, is taken to be the ratio of the dose of $\gamma$-rays, $E$, to the dose at which there is an average of 1 hit per target, $E_{0}$. That is,

$$
A=E / E_{0} .
$$

Most physical and some biological systems are 1-hit in their response to $\gamma$-rays. We have found $C$ as high as 8 in desensitized nuclear emulsions, and $m$ as high as 6 in radiobiology.

In this model of particle tracks (Katz et al., 1972) each detector is represented by experimental parameters, with no attempt to analyze mechanism. The model for physical detectors utilizes three parameters: $E_{0}$, the dose of $\gamma$-rays at which there is an average of 1 hit per target, $C$, the hittedness, and $a_{0}$, the target radius. Biological cells have greater structural complexity, for the sensitive elements and their subtargets are found within a cell nucleus (Katz, 1978). For a description of cellular response we require a fourth parameter, $\left(\sigma_{0}\right.$, which may approximate the cross-sectional area of the cell nucleus, and find it convenient to use as other parameters $m, E_{0}$, and $\kappa$, the latter being a combination of $E_{0}$ and $a_{0}$ [see equation (9)].

\section{The Cross Section}

In physical experiments the cross section represents a probability. It is only equal to the target size if the action probability is 1 whenever the projectile penetrates a target and 0 if it passes outside the target. In general the cross section can differ from the target size by orders of magnitude, either smaller or larger.

If we know the cross section, $\sigma$, the number of targets per unit volume, $N$, and the stopping power, $L$, the number of affected targets per unit pathlength, $n$, is

$$
n=\sigma N
$$

and the $G$ value (most frequently used in radiation chemistry) is, $n / L$ or

$$
G=\sigma N / L .
$$

We can look at the cross section in another way. The effects caused by heavy ions arise primarily from $\delta$-rays. If $T$ is the maximum radial distance to which $\delta$-rays penetrate, and $P(t)$ is the probability for target activation at radial distance $t$ from the ion's path, then

$$
\sigma=2 \pi \int_{t=0}^{t=T} P(t) \mathrm{d} t .
$$


The greatest possible value of the cross section is $\pi T^{2}$. This limiting value decreases as the ion slows down. The number density of $\delta$-rays increases but their maximum energy decreases (Katz and Kobetich, 1969). This effect is seen as the "thindown" of the tracks of heavy ions in electron sensitive emulsion, or as a "hook" in a plot of cross section vs. LET. Such hooks have been observed in radiobiology (Katz et al., 1985), for scintillation counters, and thermoluminescent dosimeters (Fain et al., 1980) exposed to energetic heavy ions. This effect has nothing to do with the Bragg peak in energy deposition, but is rather determined by the particle speed which sets a kinematic limit on $\delta$-ray energy.

The quantity $P(t)$ in equation (7) is determined by first calculating the radial distribution of dose from $\delta$-rays (and primary excitations and ionizations) about the path of the ion, $D(t)$, and combining that with the probability for target inactivation as a function of dose from equations (2) or (3), where the parameters $C$ (or $m$ ) and $E_{0}$ are determined from the dose response function obtained after $\gamma$-irradiation, if that information is available. Otherwise the parameters are evaluated by fitting them to experimental cross sections found after heavy ion irradiation, subject to the condition that a single set of parameters must yield all experimental cross sections found with different ions and ion speeds (Waligorski et al., 1987).

We describe tracks as being in the "grain count regime" when activated targets are rather like beads randomly placed along (and about) the ion's path. Here equation (5) is most appropriate for describing the effect. In nuclear emulsions, one counts the number of developed grains in 100 microns of path length. If activated targets make a thick track, like a hairy rope, then equation (7) is most appropriate to the track description. In nuclear emulsions one measures the optical transmission with a microdensitometer. The transmission can be related to the fraction of developed grains at different radial distances. It is only in the track width regime that we can observe thindown. Here the cross section decreases with an increase in LET. For some calculations target size plays a role. Typically we have represented the target as a short cylinder of radius $a_{0}$ whose axis is parallel to the ion's path and consider that the "dose" experienced by the cylinder is averaged over the target volume. This is because the dose falls off radially inversely with the square of the radial distance, so that the dose gradient may be large close to the ion's path, but becomes negligible at larger distances. $D(t)$ is then taken to be the average dose delivered to targets whose axis is at radial distance $t$ from the ion's path. Target size plays a minor role in calculations of the cross section for 1hit detectors, though it is somewhat more important for many-hit detectors. With 1-hit detectors measurement of the cross section gives no clear signal as to the size of the target. With many-hit detectors the cross section increases as LET to the Cth power until a plateau (which reflects the target size) is reached, marking the end of the grain count regime. Thereafter, in the track width regime the cross section increases linearly with LET until thindown takes over, when the cross section decreases with an increase in LET. These characteristics depend very much on the parameters of the detector.

\section{Detector Response to Radiation Fields}

A way to think about particle tracks in 1-hit detectors that is simpler (though less accurate) than the radial dose and the detector parameters is to imagine that a single electron (of appropriate energy) passing through a target can activate it. So also a single proton. A Chit system requires the passage of $C$ or more electrons. Poisson fluctuations in the distribution of electron paths from $\gamma$-rays are then responsible for the exponential response of 1-hit detectors to dose, and the more complex response of $C$-hit detectors. As yet we have had no further need for knowledge of the fluctuation in energy deposition, as described in microdosimetry. We must keep in mind that there are phenomena for which a detailed knowledge of fluctions is important, as in black body radiation or random noise, and that for other phenomena averages provide a sufficient description. For the Poisson distribution, the average gives us information about all other moments. To the extent that our random energy depositions can be approximated as Poissonian, detailed knowledge of the fluctuations in energy deposition is superfluous.

A many-hit response to $\gamma$-rays is nonlinear. Rather it is quadratic or of order $C$ in character. The cross section for single electron activation is zero. At increasing $\gamma$-ray or electron beam irradiation the probability that $C$ electrons pass through the target increase. It is possible to have a detector which does not even respond to fission fragments, yet which responds to high $\gamma$-ray doses. It is impossible to have a detector which responds to heavy ions which does not respond to high doses of $\gamma$-rays or electron beams (assuming that response does not arise from nuclear collisions but rather from ionizations).

For a 1-hit detector the response to dose is exponential. With a many-hit detector the situation is more complex. In the grain count regime, targets which are not activated by the passage of a single ion (because an insufficient number of $\delta$-rays passed through them) are taken to store the damage from the passage of the first particle, and may then ultimately be activated by the $\delta$ rays from second passing ion. A typical case is that of the inactivation of biological cells. Here the complexity of cell structure demands that we create a fourth parameter, related to the fact that there are many targets within a container, the cell nucleus, and that some number, $m$, of these must be inactivated in order that the cell be killed. The cross section for cellular inactivation is taken to be proportional to the cross section for the inac- 
tivation of a "quasi target" imagined to have the properties described in equation (3). We think of the cell as if it were made up like beans in a bean bag.

We calculate the cross section for inactivation of a quasi target, a single bean taken to represent the number of 1-hit beans which must be hit to inactivate the cell and whose radiosensitivity parameters are $E_{0}, a_{0}$, and $m$ for a wide range of parameter values. For these we find that the cross section increases with LET to a plateau value near the cross sectional area of the bean, as $L^{m}$ in the grain count regime at low LET, while at high LET in the track width regime the cross section varies linearly with $L$ until we enter the region of thindown where the cross section falls again. We approximate the envelope to these curves in the grain count regime by the expression

$$
P=\left[1-\exp \left(-z^{* 2} / \kappa \beta^{2}\right)\right]^{m}
$$

where $P$ is the probability for target inactivation and

$$
\kappa=E_{0} a_{0}^{2} /\left(2 \times 10^{-7} \mathrm{erg} \mathrm{cm}^{-1}\right) .
$$

We take $P$ to represent the ratio of the ion kill cross section $\sigma$ to its plateau value $\sigma_{0}$, while $z^{*}$ is the "effective charge" of an ion which may be partially clothed with electron and $\beta c$ is its speed where $c$ is the speed of light.

The fraction of intersected targets which is inactivated is $P$. These are inactivated in the ion kill mode. The fraction of targets which is intersected but not inactivated by a single ion is $(1-P)$. This residue may be inactivated in the $\gamma$-kill mode by intersecting $\delta$-rays from other ions. When a dose $D$ results from a heavy ion irradiation we take the dose fraction $P D$ to contribute to the ion kill mode of inactivation and the fraction $(1-P) D$ to contribute to the $\gamma$-kill mode of inactivation. The surviving fraction of cells irradiated by a heavy ion beam is given by equation (10), where the probability for ion kill is given by (11), and the probability for $\gamma$-kill is given by (12). We imagine that the probability for ion kill follows 1-hit statistics, and that those cells surviving the ion kill mode may be activated in the $\gamma$-kill mode, as in (12):

$$
\begin{aligned}
& N / N_{0}=\Pi_{\mathrm{i}} \Pi_{r}, \\
& \Pi_{\mathrm{i}}=\exp (-\sigma F),
\end{aligned}
$$

where $F$ is the fluence of bombarding ions and

$$
\left.\Pi_{\gamma}=1-\left\{1-\exp [-(1-P) D) / E_{0}\right]\right\} m .
$$

The equation includes four fitted parameters and gives the changing shape of survival curves with changes in the quantity $\left(z^{*} / \beta\right)^{2}$ of the bombarding ions. Once these parameters are evaluated we may extract the bean size, do, and recalculate the inactivation cross section for the particular cell line in the track width regime, and thus predict thindown. With knowledge of the secondary particle energy spectrum produced by neutrons, or other high LET irradiations (Katz and Sharma, 1973), we can calculate the surviving fraction of cells irradiated in these modalities. This four-parameter equation has been fitted to the experimental data for the survival of a large variety of cells after heavy ion irradiation as well as to cell transformation toward cancer induction, and chromosome damage. It has been used to describe heavy ion damage to photoresists, with a presumed cluster of molecules taking the place of the cell nucleus (Katz, 1983).

\section{Discussion}

These considerations have been applied to a large variety of different detecting systems, the inactivation of dry enzymes and viruses, the structure of particle tracks in emulsion, the response of scintillators and TLDs, radical production in alanine, the inactivation of biological cells, chromosome aberrations, cell transformation on heavy ion bombardment, heavy ion radiolysis of water and benzene (Katz and Huang, 1989), and damage to photoresists by heavy ion beams. We have suggested that CR-39 is a 1-hit detector because protons can produce etchable tracks, and that other track detectors must be at least 2-hit because protons do not form etchable tracks in them (Katz, 1984). This hittedness model has recently been applied to sort out data on heavy ion desorption of large organic molecules from substrate, for mass spectrometry. In some media, like water or plastics, we have had to be imaginative to suggest that there are targets, and to estimate their size. When dealing with complex radiation fields like neutron or a mixed heavy ion and $\gamma$-ray irradiation, the model makes predictive calculations possible, from knowledge of the particle-energy spectrum of all the primary and secondary heavy ions in the radiation field. We have made such calculations for biological cells inactivated by neutrons. Though there are many approximations in the model, and a total absence of mechanism in relation to the properties of the detector, it is worthy of your consideration in dealing with radiation problems, for we must first sort out the systematics of the radiation effects before undertaking a detailed study of mechanism. To apply mechanistic considerations first may lead to a confusion as to which aspects of the effects observed are dominated by track structure and which by mechanism. Such errors have been made before.

Where mechanism is the conceptual basis of an analysis, one sometimes forgets that the track physics model treats a detector as a black box in which there are initiation events and detected events, and that the intermediate interactions are not of concern. Thus, in discussing supralinearity in TLD-100, it is relevant to mechanistics that supralinearity may result from "track interactions" which take place in the "heating stage." For track physics this is simply a mechanistic explanation of why supralinearity is a 2 -hit (or 2 electron track) process. 
Finally, an appropriate goal for mechanistic models is to explain the basis of successful parametric models, and the numerical values of their parameters. In much of physics this has been the historic path. Kepler's formulation of planetary motion stimulated Newton's laws of motion and universal gravitation. Balmer's parameterization of the spectral lines of hydrogen served as the target for Bohr's model of the atom.

\section{Acknowledgement}

This work is supported by the United States Department of Energy.

\section{References}

Dertinger H. and Jung H. (1970) Molecular Radiation Biology. Springer, New York.

Fain J., Montret M., and Sahraoui L. (1980) Thermoluminescent response of $\mathrm{CaF}_{2}$ :Dy and $\mathrm{LiF}: \mathrm{MgTi}$ under heavy ion bombardment. Nucl. Instrum. Meth. 175, 37.

Hamm R. N., Wright H. A., Katz R., Turner J. E., and Ritchie R. H. (1978) Calculated yields and slowing-down spectra for electrons in liquid water: implications for electron and photon RBE. Phys. Med. Biol. 23, 1149.

ICRU (1983) Microdosimetry. ICRU Report 36, International Commission of Radiation Units and Measurements.
Katz R. (1978) Track structure theory in radiobiology and in radiation detection. Nucl. Track Detection 2, 1.

Katz R. (1983) Track structure interpretation of heavy ion lithography in relation to radiobiology and to the structure of etchable tracks in solid state nuclear track detectors. 8th Symp. Microdosimetry, Commission of the European Communities, p. 135.

Katz R. (1984) Formation of etchable tracks in plastics. Nucl. Tracks Radiat. Meas. 8, 1.

Katz R. and Huang G.-R. (1989) Track core effects in heavy ion radiolysis. Radiat. Phys. Chem. 33, 345.

Katz R. and Kobetich E. J. (1969) Particle tracks in emulsion. Phys. Rev. 186, 344.

Katz R. and Sharma S. C. (1973) Response of cells to fast neutrons, stopped pions, and heavy ion beams. Nucl. Instrum. Meth. 110, 93.

Katz R., Sharma S. C., and Homayoonfar M. (1972) The structure of particle tracks. In: Topics in Radiation Dosimetry (Edited by Attix F. H.). Academic Press, New York.

Katz R., Dunn D. E., and Sinclair G. L. (1985) Thindown in radiobiology. Radiat. Prot. Dosimetry 13, 281.

Suntharalingam N. and Cameron J. R. (1969) Thermoluminescent response of LiF to radiations with different LET. Phys. Med. Biol. 14, 397.

Waligorski M. P. R., Hamm R. N., and Katz R. (1986) The radial distribution of dose around the path of a heavy ion in liquid water. Nucl. Tracks Radiat. Meas. 11, 309.

Waligorski M. P. R., Loh K. S., and Katz R. (1987) Inactivation of dry enzymes and viruses by energetic heavy ions. $R a$ diat. Phys. Chem. 30, 201. 